\title{
ORIGINAL
}

\section{TENDENCIA EN LA UTILIZACIÓN DE ANTIAGREGANTES EN LA COMUNIDAD VALENCIANA (2000-2005)}

\author{
Beatriz Román Llamosí (1), Rocío Broseta Solaz (2), Joan Quiles Izquierdo (3) y Amalia Úbeda \\ Pascual (1)
}

(1) Departamento Farmacología. Universitat de Valencia (UVEG). España.

(2) Oficina de Gestión Abucasis. Sistema Gaia. Agencia Valenciana de Salud. Conselleria de Sanitat. Generalitat Valenciana. España.

(3) Dirección General de Salud Pública. Conselleria de Sanitat. Generalitat Valenciana. España.

\section{RESUMEN}

Fundamento: Los antiagregantes plaquetarios son fármacos básicos para la prevención de enfermedades isquémicas arteriales. El objetivo de este trabajo es conocer la evolución de su utilización en Atención Primaria en la Comunidad Valenciana durante el periodo 2000-2005.

Métodos: Estudio descriptivo del consumo de los antiagregantes plaquetarios (código ATC: B01AC) dispensados con cargo al Sistema Nacional de Salud en la Comunidad Valenciana en el ámbito de Atención Primaria. Datos expresados en dosis diarias definidas (DDD) por 1.000 habitantes y día.

Resultados: En 2005 tres fármacos representaron el 98\% de la prescripción global (ácido acetilsalicílico (AAS) $66 \%$, clopidogrel $23 \%$ y triflusal $9 \%$ ). El consumo de antiagregantes orales se incrementó en un $23 \%$ entre 2000 y 2005 (desde 29,6 DDD/1.000 hab y día hasta 36,5). El clopidogrel mostró un incremento del $218 \%$, mientras que el ácido acetilsalicílico fue el principio de mayor consumo con una cuota de utilización bastante estable durante todo el periodo (próxima al $70 \%$ ). El gasto generado por este grupo de fármacos se duplicó, el clopidogrel fue el fármaco con mayor coste/DDD $(2,14 €)$, en 2005 su utilización constituyó un $23 \%$ del total de antiagregantes pero su gasto representó el $76 \%$ del total.

Conclusiones: En este periodo se incrementó el consumo de antiagregantes en la Comunidad Valenciana. El AAS mantuvo estable su utilización, mientras que el clopidogrel aumentó su cuota de mercado pese a las recomendaciones de las guías terapéuticas y las restricciones para su uso. El consumo del clopidogrel contribuyó de forma notable al gasto farmacéutico de este grupo.

Palabras clave: Antiagregantes plaquetarios. Clopidogrel. Ácido acetilsalicícico. Utilización de medicamentos.

Correspondencia:

Dra. Amalia Úbeda

Dpt. Farmacología.

Universitat de Valencia. UVEG.

Avda. Vicent Andres Estelles, sn. 46100 Burjassot. Valencia

Correo electrónico: Amalia.Ubeda@uv.es

\section{ABSTRACT \\ Trend in Platelet Antiaggregants Utilization in the Autonomous Community of Valencia, Spain (2000-2005)}

Background: Platelet antiaggregants are basic drugs for preventing ischemic arterial diseases. This study is aimed at ascertaining the trend in their use in Primary Care in the Autonomous Community of Valencia during the 2000-2005 period.

Methods: Descriptive study of the use of platelet antiaggregants (ATC code: B01AC) dispensed charged to the National Health System in the Autonomous Community of Valencia in Primary Care. Data given in defined daily doses (DDD) per 1,000 inhabitants per day.

Results: In 2005, three drugs totalled $98 \%$ of all those prescribed overall (acetyl salicylicacid (ASA) 66\%, clopidogrel $23 \%$ and triflusal $9 \%$ ). Oral antiaggregant use rose by $23 \%$ within the 2000-2005 period (from 29.6 to 36.5 DDD/1,000 inhab./day). Clopidogrel showed a $218 \%$ increase, whilst ASA was the most used drug, with quite a stable percentage of use throughout said time period (nearing 70\%). The expense generated by this group of drugs doubled, clopidogrel having been the highest-cost drug/DDD (2.14 EUROS), its use having totalled $23 \%$ of all antiaggregants yet the expense thereof having totalled $76 \%$ of the total expenditure.

Conclusions: The use of antiaggregants increased in the Autonomous Community of Valencia during the time period under study. The utilization of ASA remained stable, whilst clopidogrel increased its market share despite the treatment guide recommendations and the restrictions on its use. The consumption of clopidogrel noticeably contributed to the drug spending for this group.

Key words: Platelet aggregation inhibitors, Clopidogrel. Aspirin. Drug utilizatión. 


\section{INTRODUCCIÓN}

Las enfermedades cardiovasculares constituyen la primera causa de mortalidad en los países occidentales. En España, estas patologías representaron casi el $34 \%$ de todas las causas de defunción en el año 2003, destacando la mortalidad por accidentes cerebrovasculares en las mujeres $(11,9 \%)$ y por cardiopatía isquémica en varones $(11,5 \%)$. También suponen una de las principales causas de hospitalización, así el $12,9 \%$ de las altas hospitalarias se atribuyen a enfermedades del sistema circulatorio ${ }^{1}$. La prevalencia de las enfermedades cardiovasculares es baja antes de los 55 años, pero aumenta progresivamente con la edad, especialmente en varones ${ }^{2}$. La prevención y tratamiento de las enfermedades cardiovasculares supone importantes repercusiones socioeconómicas.

En la actualidad, el tratamiento antitrombótico constituye una de las piedras angulares en la prevención y tratamiento de los accidentes isquémicos. En concreto, los antiagregantes plaquetarios tienen un papel fundamental en la prevención secundaria de enfermedades vasculares periféricas, así como cardio y cerebrovasculares. Así mismo, distintas guías recomiendan el uso de antiagregantes para la prevención primaria en grupos de alto riesgo $0^{3,4}$.

En los últimos años se ha producido un incremento en la utilización de los fármacos antiagregantes plaquetarios, evidenciado por los datos de consumo del Sistema Nacional de Salud (SNS). Así en 2003 este grupo representaba el décimo con mayor coste para el SNS (a través de receta oficial), siendo el principio activo clopidogrel el tercer fármaco más utilizado en 2004 (según su importe) $)^{5,6}$. Los estudios realizados en España sobre la utilización de antiagregantes recogen datos anteriores al 1998, por lo que no incluyen a clopidogrel ${ }^{7,8}$. El objetivo del presente trabajo es analizar la evolución del consumo de los antiagregan- tes plaquetarios en Atención Primaria en el sector público sanitario de la Comunidad Valenciana durante el periodo 2000-2005.

\section{MATERIAL Y MÉTODOS}

Estudio descriptivo de las prescripciones de inhibidores de la agregación plaquetaria, excluida heparina (grupo terapéutico B01AC) y los fármacos de uso parenteral, durante el periodo 2000-2005 en el ámbito extrahospitalario de la Comunidad Valenciana. Los datos de consumo y costes económicos han sido obtenidos a través de la Consellería de Sanitat utilizando como fuente el sistema gestor de la prestación farmacéutica GAIA, que recoge las especialidades farmacéuticas dispensadas con recetas en oficinas de farmacia, con cargo a la SNS. Los datos de población asegurada también fueron aportados por esta Consellería. Asumimos que la dispensación equivale a utilización, y que esta dispensación se considera una aproximación aceptable del consumo total, ya que el $98 \%$ de la población española está incluida en el SNS y estos medicamentos se consumen mayoritariamente con receta.

Los datos se expresan en dosis diarias definidas (DDD), siguiendo las recomendaciones establecidas por la OMS, que permiten transformar el número de envases consumidos en DDD consumidas anualmente. Para obtener el consumo en DDD/ 1.000 habitantes y día, se dividen las DDD consumidas por la población adscrita al SNS en la Comunidad Valenciana y los días del año y se multiplica por $1.000^{9}$.

Los principios activos considerados en el análisis han sido: Ácido acetilsalicílico (AAS), triflusal, dipiridamol, ticlopidina, clopidogrel, carbasalato, ditazol y la asociación AAS-dipiridamol. Los datos de consumo de carbasalato, ditazol y la asociación AASdipiridamol, computan en el consumo global pero no se han detallado en los resultados ya que presentan valores inferiores a 0,5 DDD/ 
1.000 hab. y día. Tras calcular las DDD/ 1.000 hab. y día de cada fármaco se obtuvo el porcentaje de utilización sobre el total del grupo. Se calculó el importe anual por principio activo y su porcentaje respecto al total del grupo terapéutico. Se han estimado los costes totales en euros corrientes del año considerado y en euros constantes corrigiendo los datos con la inflación en base al año 2000 (Indice de Precios de Consumo general obtenido del Instituto Nacional de Estadística ${ }^{10}$.

Los incrementos en el consumo se calcularon restando el valor de las DDD/ 1.000 hab. y día del año 2005 al valor del año 2000. Esta diferencia se divide por el valor del año 2000 y se expresa en porcentaje.

\section{RESULTADOS}

La utilización de antiagregantes en atención primaria (uso no hospitalario) se incrementó en la Comunidad Valenciana durante el periodo considerado, pasando de 29,6 DDD/ 1.000 hab. y día en el año 2000 a 36,5 en el 2005 (incremento del 23\%). En los tres últimos años se estabilizó el consumo, incluso con una ligera reducción en 2005. La tabla 1 muestra la evolución en el consumo de los cinco principios más empleados. El AAS fue el antiagregante de mayor consumo, aunque en los últimos años las DDD/ 1.000 hab. y día presentaron valores semejantes. Clopidogrel presentó un continuo incremento en su consumo.

El consumo de AAS se incrementó desde el 2000 hasta el 2005 en un $21 \%$. Sin embargo, si analizamos su porcentaje de utilización o cuota de mercado, se observa un estancamiento entre 66-70\% durante estos años (figura 1). Clopidogrel,

\section{Figura 1}

Evolución del porcentaje de utilización de los fármacos antiagregantes respecto al consumo total

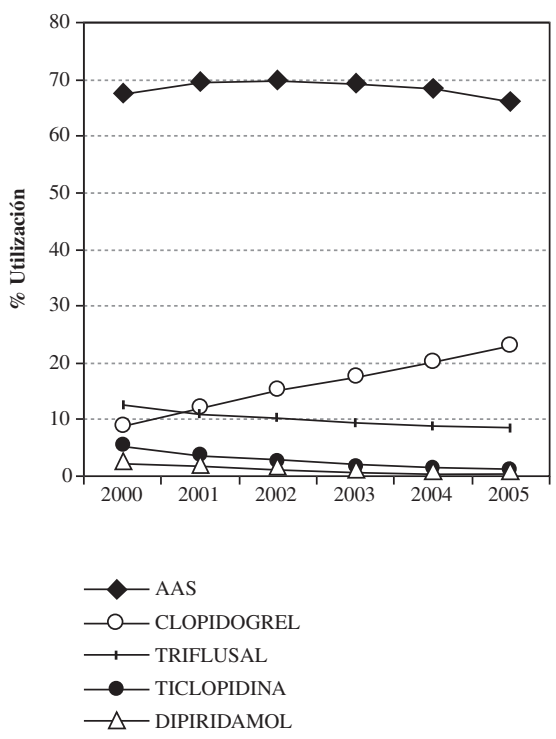

Tabla 1

Evolución de las DDD/1.000 hab y día de los antiagregantes plaquetarios en la Comunidad Valenciana durante el periodo 2000-2005

\begin{tabular}{|l|c|c|c|c|c|c|c|}
\hline & $\mathbf{2 0 0 0}$ & $\mathbf{2 0 0 1}$ & $\mathbf{2 0 0 2}$ & $\mathbf{2 0 0 3}$ & $\mathbf{2 0 0 4}$ & $\mathbf{2 0 0 5}$ & Incremento* \\
\hline AAS & 20,00 & 23,83 & 24,94 & 26,12 & 25,91 & 24,16 & $21 \%$ \\
\hline Triflusal & 3,76 & 3,80 & 3,75 & 3,63 & 3,42 & 3,20 & $-15 \%$ \\
\hline Clopidogrel & 2,64 & 4,15 & 5,47 & 6,66 & 7,67 & 8,39 & $218 \%$ \\
\hline Ticlopidina & 1,65 & 1,29 & 0,91 & 0,69 & 0,49 & 0,40 & $-76 \%$ \\
\hline Dipiridamol & 0,78 & 0,68 & 0,62 & 0,48 & 0,38 & 0,31 & $-60 \%$ \\
\hline Total & $\mathbf{2 9 , 6 3}$ & $\mathbf{3 4 , 3 0}$ & $\mathbf{3 5 , 7 4}$ & $\mathbf{3 7 , 6 2}$ & $\mathbf{3 7 , 8 7}$ & $\mathbf{3 6 , 5 0}$ & $\mathbf{2 3 \%}$ \\
\hline
\end{tabular}

DDD: dosis diarias definidas/1.000 habitantes y día. Los valores de los fármacos: carbasalato cálcico, ditazol y la asociación AAS-dipiridamol, no se incluyen por ser inferiores a $0,5 \mathrm{DDD} / 1.000$ habitantes y día, pero si se consideran en el computo total.

*El incremento se ha calculado con los valores de 2005 y 2000. 


\section{Figura 2}

Evolución del coste (euros corrientes) de clopidogrel, AAS, triflusal y el total de antiagregantes en la Comunidad Valenciana

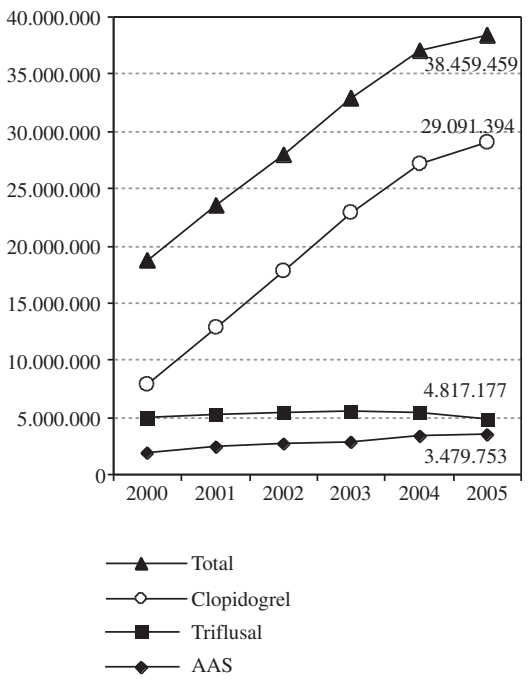

principio comercializado en 1999, alcanzó un elevado incremento en consumo $(218 \%)$, siendo el segundo antiagregante más empleado desde 2001. Ticlopidina y dipiridamol redujeron su porcentaje de utilización (valores de incremento negativos). La DDD/1.000 hab. y día de triflusal también se redujo (-15\%), con un descenso mayor en la cuota de mercado $(-31 \%)$. En el año 2005, tres antiagregantes (AAS, clopidogrel y triflusal) representaban el
98\% del total de las DDD/1.000 hab. y día prescritas.

El gasto total de este grupo se duplicó en estos años, pasando de 18 millones a más de 38 millones de euros corrientes. El gasto en 2005 corrigiendo la inflación fue de 32,8 millones de euros constantes. En la Figura 2 se observa como el aumento en el consumo de clopidogrel contribuyó directamente en el incremento total del gasto $(75,6 \%$ del total del coste en 2005). En 2005 el tratamiento con clopidrogrel resulta 23,8 veces más caro que con el fármaco de referencia del grupo AAS (Tabla 2). Clopidogrel es desde 2000 el fármaco con mayor gasto por tener el coste/DDD más elevado. Durante este periodo triflusal ha contribuido con un gasto superior a AAS, pese a representar aproximadamente un $10 \%$ de la cuota de mercado.

La proporción de coste que representa cada principio activo se muestra en la figura 3. Clopidogrel es el principio más caro y el incremento de su consumo supone una proporción muy elevada del incremento en los costes. AAS fue el antiagregante más consumido (66-70\%), sin embargo sólo representó el $9-10 \%$ del gasto total del grupo; ello se debe a su menor coste por DDD (Tabla 2). La proporción coste/DDD de clopidogrel y ticlopidina son las más elevadas. Considerando el porcentaje de utilización, y con la simplificación de que los antiagregantes se usan exclusivamente en

Tabla 2

Datos de utilización de los principales antiagregantes y coste en 2005 en la Comunidad Valenciana

\begin{tabular}{|l|c|c|c|c|c|}
\hline \multicolumn{1}{|c|}{ Principio Activo } & DDD* & DDD dispensadas & Coste (euros) & \% Coste Total & Coste/DDD (euros) \\
\hline AAS & $1 \mathrm{comp}$ & 39.075 .988 & 3.479 .753 & $9,0 \%$ & 0,09 \\
\hline Triflusal & $600 \mathrm{mg}$ & 5.175 .490 & 4.817 .177 & $12,5 \%$ & 0,93 \\
\hline Clopidogrel & $75 \mathrm{mg}$ & 13.568 .660 & 29.091 .394 & $75,6 \%$ & 2,14 \\
\hline Ticlopidina & $500 \mathrm{mg}$ & 642.660 & 834.411 & $2,2 \%$ & 1,30 \\
\hline Dipiridamol & $400 \mathrm{mg}$ & 502.611 & 207.137 & $0,5 \%$ & 0,41 \\
\hline Total & & $\mathbf{5 9 . 0 2 6 . 1 8 9}$ & $\mathbf{3 8 . 4 5 9 . 4 5 9}$ & & $\mathbf{0 , 6 5}$ \\
\hline
\end{tabular}

* DDD: dosis diarias definidas, valores establecidos por la OMS (9).

DDD dispensadas: dosis diarias definidas dispensadas en 2005 en la Comunidad Valenciana. 


\section{Figura 3}

Evolución del porcentaje de coste de cada fármaco respecto al total de gasto en antiagregantes en la Comunidad Valenciana

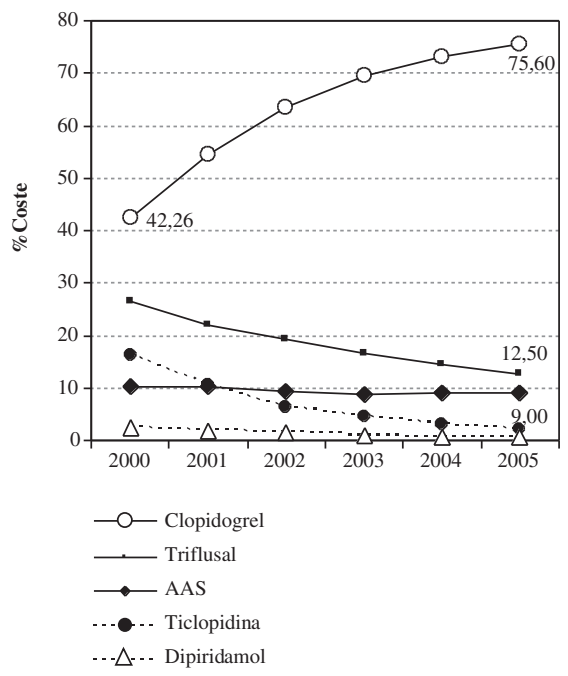

monoterapia (aunque a veces se aconseja su asociación), se podría decir que en 2005 clopidogrel fue el fármaco que utilizó alrededor de un $23 \%$ del total de pacientes en tratamiento con antiagregantes orales representando el $76 \%$ del gasto total de este grupo en 2005. El coste/DDD de clopidogrel en 2005 se redujo respecto al de 2004, así en Marzo de 2005 el coste real pasó de $2,24 €$ a $2,13 €$, lo cual reduce la pendiente de la evolución anual del coste (figura 2).

\section{DISCUSIÓN}

Los datos de este estudio muestran un incrementó del consumo de antiagregantes plaquetarios en la Comunidad Valenciana, lo cual concuerda con la mayor preocupación actual por la prevención secundaria de las enfermedades cardiovasculares y las mayores evidencias de su eficacia y el balance beneficio/riesgo favorable en pacientes de alto riesgo indicadas en las guías más recientes ${ }^{3,4}$.
Desde los años 90 se observa un incremento en el consumo de antiagregantes en España, con un ascenso de AAS y un descenso de dipiridamol ${ }^{7}$. Nuestros datos indican que AAS fue el antiagregante más consumido desde 2000, lo cual está en consonancia con las recomendaciones del Documento Consenso de Expertos sobre el uso de antiplaquetarios, que lo considera de primera elección para la profilaxis cardiovascular $^{3}$. Sin embargo, se produjo un estancamiento relativo de su utilización en los últimos años en la Comunidad Valenciana, mientras que clopidogrel incrementó considerablemente su cuota de mercado. Si el incremento observado del consumo global de antiagregantes se relaciona con mayores necesidades de la población y una mayor prescripción y uso en prevención secundaria, sería más lógico observar un crecimiento paralelo en la utilización de AAS, lo cual no ocurre, y sugiere que clopidogrel está en parte sustituyendo a AAS inadecuadamente.

Clopidogrel se autorizó en España en 1999, basándose en un ensayo comparativo de seguridad y eficacia versus AAS en la prevención secundaria de eventos ateroscleróticos (CAPRIE) ${ }^{11}$. Según este estudio, habría que tratar a 196 pacientes durante un año con clopidogrel en lugar de con AAS para evitar un evento aterosclerótico adicional. Pese a que clopidogrel mostró una eficacia ligeramente superior a AAS en la prevención de eventos isquémicos vasculares en este estudio, las guías farmacoterapéuticas continúan recomendando al AAS como la opción más coste-efectiva en prevención secundaria de sucesos cardiovasculares, mientras que clopidogrel estaría indicado únicamente en pacientes que no toleran $\mathrm{AAS}^{3,12}$.

Según datos del estudio CAPRIE, clopidogrel presentó una menor incidencia de hemorragia gastrointestinal severa que AAS $(0,49 \%$ frente a $0,71 \%)$, sin que se observaran diferencias estadísticamente significati- 
vas en otros efectos adversos severos como: molestias gastrointestinales o trastornos hemorrágicos en general. Por otra parte, clopidogrel presentó más casos de rash severo que AAS. Esta "mejor tolerancia" gastrointestinal de clopidogrel sugiere que su empleo sería ventajoso en pacientes con riesgo de úlceras por AAS, sin embargo recientemente se ha descrito que en pacientes con historial de úlceras sangrantes causadas por AAS, el tratamiento con AAS+esomeprazol presenta una menor incidencia de recurrencia de la úlcera que el uso de clopidogre ${ }^{13}$. Asimismo, un estudio de ingresos hospitalarios confirma que el riesgo de hemorragias gastrointestinales es menor en la asociación AAS+ un inhibidor de la bomba de protones que con clopidogre $^{14}$. Estos estudios no apoyan la habitual recomendación de emplear clopidogrel como alternativa a AAS en pacientes con riesgo hemorrágico gastrointestinal. Además, se ha demostrado que la asociación AAS más esomeprazol u omeprazol es más coste-efectiva que clopidogrel para el SNS español ${ }^{15}$.

La comercialización de clopidogrel ha influido también en la reducción de la utilización de la otra tienopiridina ticlopidina. Según un estudio de 1998, el empleo de ticlopidina era excesivo e inadecuado en muchos $\operatorname{casos}^{8}$, además este fármaco puede causar toxicidad hematológica, por lo que requiere un seguimiento estricto en los primeros meses del tratamiento. Clopidogrel presenta un menor riesgo hematológico que ticlopidina, sin requerir un control especial $^{16}$, y es lógica esta sustitución, pero probablemente el uso de clopidogrel también sea excesivo e inadecuado.

El incremento del consumo de clopidogrel puede deberse, al menos en parte, a su uso asociado a AAS, indicación aprobada en septiembre de 2002 por la EMEA. Así según la ficha técnica (o RCP) ${ }^{17}$ de 2005: “clopidogrel está indicado en prevención de eventos aterotrombóticos en:
- Pacientes que han sufrido infarto de miocardio (desde los pocos días hasta un máximo de 35 días), infarto cerebral (desde los 7 días hasta un máximo de 6 meses) o arteriopatía periférica establecida.

- Pacientes que presentan síndrome coronario agudo sin elevación del segmento ST (angina inestable o infarto de miocardio sin onda Q), en combinación con ácido acetilsalicílico (AAS)."

Posteriormente, en 2006, se amplia la indicación de su uso asociado a AAS a "pacientes que presentan síndrome coronario agudo: Infarto de miocardio agudo con elevación del segmento ST, en combinación con AAS en pacientes tratados médicamente de elección para terapia trombolítica"17.

El posible beneficio terapéutico de clopidogrel (empleado solo o asociado a AAS) en la prevención de distintos procesos aterotrombóticos se ha evaluado en los últimos años dando lugar a numerosos artículos ${ }^{16}$, que pueden haber contribuido a promover su prescripción frente al resto de antiagregantes plaquetarios. La promoción comercial de las nuevas evidencias aportadas por ensayos clínicos se considera el factor principal para modificar la práctica clínica, mientras que su mera publicación tan sólo repercute en pequeños cambios en la práctica clínica, como demuestra el artículo de Majumdar y cols. $(2003)^{18}$. Esta promoción tiende a destacar los resultados más convenientes para la industria farmacéutica, como se ha observado para el clopidogrel tras el ensayo CHARISMA ${ }^{19}$.

Los resultados de nuestro estudio, basado en datos agregados de consumo, no permiten cuantificar ni valorar el uso asociado de clopidogrel a AAS, aunque un análisis mensual más pormenorizado (datos no mostrados) no evidenció que hubiera un mayor incremento en la tendencia de consumo de clopidogrel a partir de septiembre de 2002 respecto al periodo anterior. 
Según un estudio hospitalario de prescripción-indicación, clopidogrel es empleado en distintos pacientes para indicaciones o durante un tiempo que no concuerda con las recomendaciones de las guías clínicas. Así, clopidogrel es empleado en monoterapia en numerosos pacientes sin uso previo de AAS y sin que AAS este contraindica$\mathrm{do}^{20}$. En nuestro ámbito, la prescripción a nivel hospitalario o por especialistas puede llevar a su uso en situaciones donde AAS sería de primera elección o bien alargar su tratamiento por un tiempo superior al de sus indicaciones terapéuticas, ya que la continuación de su prescripción se realiza en Atención Primaria de forma inducida.

El tercer antiagregante de mayor consumo en la Comunidad Valenciana fue triflusal. Datos nacionales indican que hasta 1993 era el antiagregante más empleado, y en 1998 supuso un 23\% del total de antiagregantes consumidos ${ }^{8}$. Durante el periodo estudiado se redujo su consumo aunque significó en 2005 casi el 9\% del total de los antiagregantes. Triflusal, ha mostrado eficacia semejante a AAS en prevención secundaria en algunos grupos de pacientes con elevado riesgo cardiovascular (tras accidente cerebrovascular isquémico y en infarto agudo de miocardio), con un menor riesgo de hemorragias ${ }^{21}$. Asimismo, un reciente estudio epidemiológico también corrobora las menores tasas de complicaciones hemorrágicas respecto a otros antiagregantes ${ }^{14}$. Sin embargo, el documento de consenso Europeo no recomienda su uso por las limitadas pruebas de su eficacia ${ }^{3}$. En la valoración de su utilización como alternativa a AAS, también convendría considerar que triflusal tiene un coste/DDD 12 veces superior a AAS. Así, la hipotética sustitución del consumo de triflusal por AAS en 2005, supondría un ahorro de más de 4,3 millones de euros en la Comunidad Valenciana.

Según los datos de DDD/ 1.000 hab. y día de 2005, y considerando que estos fármacos suelen emplearse en tratamientos crónicos y en monoterapia, podemos inferir que 36 personas de cada 1.000 habitantes toman un antiagregante cada día. La aplicación de la fórmula DDD/1.000 hab. y día presenta un sesgo importante, ya que considera toda la población asegurada (4,3 millones), mientras que las patologías cardiovasculares tienen una mayor prevalencia en la edad adulta y sobre todo a partir de 65 años $^{2}$. Si estimamos la DDD/1.000 hab. y día considerando exclusivamente la población mayor de 65 años (el 17\% de la población en la Comunidad Valenciana), la cifra aproximada de usuarios diarios de antiagregantes sería del 21\% (215 DDD/1.000 habitantes mayores de 65 años y día). Este valor está en el mismo rango que en Suecia donde alrededor del $27 \%$ de habitantes de 60 o más años utiliza fármacos antitrombóticos ${ }^{22}$.

Para explicar el crecimiento del consumo de antiagregantes plaquetarios se deben considerar distintos factores:

- El aumento de la prevalencia de enfermedades vasculares donde estos fármacos están indicados, lo cual se relaciona tanto con el envejecimiento de la población, como con una mayor incidencia de síndromes coronarios agudos recurrentes (consecuencia de la menor letalidad) y un número creciente de intervenciones coro$\operatorname{narias}^{23}$.

- Mayor proporción de población incluida dentro del grupo considerado de alto riesgo cardiovascular, que requieren prevención de eventos aterotrombóticos. Así en los últimos años, se ha debatido sobre la idoneidad de incluir a todos los diabéticos (mayores de 40 años) en la prevención primaria con aspirina, aunque una revisión reciente considera que no existen suficientes pruebas científicas para sustentar esta indicación ${ }^{24}$. La guía europea de prevención cardiovascular lo recomienda solo cuando existen otros factores de riesgo $\operatorname{asociados}^{4,25}$. 
- Mayor sensibilización del profesional médico para la prescripción de estos fármacos como prevención secundaria ante la publicación de estudios que han alertado sobre un deficiente uso de medicación cardioprotectora en prevención secundaria de cardiopatía isquémi$\mathrm{ca}^{26,27}$.

El consumo de los antiagregantes plaquetarios ha aumentado considerablemente en los últimos cinco años en la Comunidad Valenciana y también a nivel nacional, aspecto que puede haber contribuido a la reducción del índice de mortalidad ajustado por edad tanto por enfermedades isquémicas del corazón como por enfermedad cerebrovascular $^{28}$. Pese a ello, algunos estudios epidemiológicos indican que no todos los pacientes que podrían beneficiarse de una terapia antiagregante están recibiendo tratamiento $26,29,30$.

El constante incremento en el consumo de clopidogrel plantea una reflexión sobre su adecuada utilización, ya que su empleo en prevención secundaria de enfermedades cardiovasculares se considera una alternativa en los pacientes que no toleran $\mathrm{AAS}^{3,12}$ y su indicación en combinación con AAS es muy concreta, sin que este claramente establecida la duración de estos tratamientos ${ }^{12,16}$. Además, el coste de este fármaco es muy elevado y presenta un gran impacto en el gasto farmacéutico. Son necesarios estudios de prescripciónindicación para conocer las situaciones clínicas en las que se está utilizando clopidogrel en la práctica ambulatoria, analizando la prescripción inducida hospitalaria y la duración de los tratamientos, para poder corregir posibles desviaciones de las guías terapéuticas, e intervenir para ajustar su empleo a las situaciones clínicas donde realmente supone un beneficio respecto al uso de AAS; ello contribuiría al uso racional de estos medicamentos y a conseguir una mejor distribución de los recursos sanitarios disponibles.

\section{BIBLIOGRAFÍA}

1. Defunciones según la causa de muerte 2003. Encuesta de morbilidad hospitalaria 2003. INEbase. [citado 1 de sept 2006]. www.ine.es/prodyser/catalogo/sanitari.htm.

2. Baena JM. Del Val JL, Tomás J, Martínez JL, Martín R. González I, y cols. Epidemiología de las enfermedades cardiovasculares y factores de riesgo en atención primaria. Rev Esp Cardiol. 2005;58:367-73.

3. Patrono C, Bachmann F, Baigent C, Bode C, De Caterina R, Charbonnier B y cols. Grupo de Trabajo sobre el uso de agentes antiplaquetarios en pacientes con enfermedad cardiovascular aterosclerótica de la Sociedad Europea de Cardiología. Documento de Consenso de Expertos sobre el uso de agentes antiplaquetarios. Rev Esp Cardiol. 2004; 57:963-80.

4. Tercer grupo de trabajo de las Sociedades Europeas y otras Sociedades sobre Prevención Cardiovascular en la Práctica Clínica. Guía Europea de Prevención Cardiovascular en la Práctica Clínica. Rev Esp Salud Pública. 2004;78:439-56.

5. Grupos terapéuticos y principios activos de mayor consumo en el sistema nacional de salud durante 2003. Inf Ter Sist Nal Salud. 2004;28:121-4.

6. Grupos terapéuticos y principios activos de mayor consumo en el sistema nacional de salud durante 2004. Inf Ter Sist Nal Salud. 2005;29:49-53.

7. Plata Alonso AM, Blanco Montagut LE. Evolución de la utilización de Anticoagulantes e Inhibidores Agregación Plaquetaria (B01) en el Área de Salud de Âvila, mediante DDD (Dosis Diarias Definidas). Centro de Salud. 1998;6:42-9.

8. Del Pino AM, De Abajo FJ, Montero D, Madruga M, Martín Serrano G. Estudio de utilización de ticlopidina en oficinas de farmacia de España. Med Clin (Barc). 2000; 115:211-3.

9. Guidelines for ATC classification and DDD assignment. Oslo: WHO Collaborationg Centre for Drug Statistics Methodology; 2001. [citado 1 de sept 2006]. http://www.whocc.no/atcddd/indexdatabase/index.php

10. Indice de precios de consumo. Instituto Nacional de Estadística. [citado 1 de sept 2006]. www.ine.es/daco/ipc.htm.

11. CAPRIE Steering Comité. A randomised, blinded, trial of clopidogrel versus aspirin in patients at 
risk of ischaemic events (CAPRIE). Lancet. 1996;438:1329-39.

12. Antiagregación en atención primaria. Respondiendo preguntas. INFAC. 2004;12:29-34. [citado 1 de sept 2006]. www.euskadi.net/sanidad/cevime.

13. Chan FKL, Ching JYL, Hung LCT, Wong VWS, Leung VKS, Kung NNS y cols. Clopidogrel versus aspirin and esomeprazole to prevent recurrent ulcer bleeding. N Engl J Med. 2005; 352: 238244.

14. Ibáñez L, Vidal X, Vendrell L, Moretti U, Laporte JR. Spanish-Italian Collaborative Group for the Epidemiology of Gastrointestinal Bleeding. Upper gastrointestinal bleeding associated with antiplatelet drugs. Aliment Pharmacol Ther. 2006;23:235-42.

15. Piñol C. Evaluación económica del tratamiento con ácido acetilsalicílico más esomeprazol comparado con clopidogrel en la prevención de la hemorragia gastrointestinal. Gac Sanit. 2006;20:54-8.

16. Plosker GL, Lyseng-Williamson KA. Clopidogrel: A Review of its Use in the Prevention of Thrombosis. Drugs. 2007; 67:613-46.

17. EMEA. Plavix ®. Resumen de las características del producto. [citado 2 de Marzo 2007] http://www.emea.eu.int/humandocs/Humans/EPA R/plavix/plavix.htm..

18. Puig Junoy J. La promoción comercial es lo más efectivo para transferir la evidencia científica a la práctica clínica. [Majumdar SR, McAlister FA, Soumerai SB. Synergy between publication and promotion: Comparing adoption of new evidence in Canada and the United States. Am J Med 2003; 115:467-72.] Gestión clínica y sanitaria. 2004;6:30. http://www.iiss.es/gcs/.

19. Moliner J. Sin embargo, el clopidogrel resulta perjudicial para quienes únicamente tienen factores de riesgo. [Bhatt DL, Fox KA, Hacke W et al. del grupo CHARISMA. Clopidogrel and aspirin versus aspirin alone for the prevention of atherotrombotic events. N Engl J Med. 2006; 354:1706-17]. Gestión clínica y sanitaria. 2006;8:51. http://www.iiss.es/gcs/.

20. Kubler PA, Pillans PI, Marrinan MC, Frogley M. Concordance between clopidogrel use and prescribing. Inter Med J. 2004;34:663-7.
21. Costa J, Ferro JM, Matias-Guiu J, Alvarez-Sabin J, Torres F. Triflusal para la prevención de eventos vasculares graves en personas de alto riesgo (Revisión Cochrane traducida). En: La Biblioteca Cochrane Plus, Número 1. Oxford: Update Software Ltd; 2007. Disponible en: http://www.update-software.com.

22. Silwer L, Lundborg CS. Patterns of drug use during a 15 year period: data from a Swedish county, 1988-2002.Pharmacoepidemiol Drug Saf. 2005;14:813-20.

23. Marrugat J, Elosua R, Martí H. Epidemiología de la cardiopatía isquémica en España: estimación del número de casos y de las tendencias entre 1997 y 2005. Rev Esp Cardiol. 2002;55:337-46.

24. Maciá-Bobes C, Monzón-Fernández A, Fernández-García E. La prevención primaria con aspirina de enfermedades cardiovasculares en personas diabéticas. Revisión de las pruebas disponibles. Rev Esp Salud Pública. 2006;80:613-20.

25. Balaguer-Vintró I. Prevención primaria, aspirina y diabetes. Rev Esp Salud Pública. 2006;80:609-12.

26. Grupo PRESENCIAP Estudio de prevención secundaria de la cardiopatía isquémica en la atención primaria (PRESENCIAP). Aten Primaria. 2001;27:29-32.

27. Velasco JA, Cosín J, López-Sendón JL, de Teresa E, de Oya M, Selles G. Nuevos datos sobre la prevención secundaria del infarto de miocardio en España. Resultados del estudio PREVESE II. Rev Esp Cardiol. 2002;55:801-9.

28. Grupo de trabajo de la conferencia de presidentes para el análisis del gasto sanitario. Informe para el análisis del gasto sanitario. Ministerio de Economía y Hacienda. Julio 2005. [citado 1 de sept 2006]. www.msc.es/estadEstudios/estadisticas/sisInfSanSNS/pdf/IGTGS2005.pdf.

29. López de la Iglesia J, Escudero Alvarez S, González García AM, Mencia Mieres A, García Andrés LE, Moran Fernández B. Empleo de antiagregantes en la prevención primaria y secundaria cardiovascular del diabético en el medio urbano y rural del área de León. Aten Primaria. 2003;31:361-5.

30. Esmatjes E, Castell C, Franch J, Puigoriol E, Hernáez R. Consumo de ácido acetilsalicílico en pacientes con diabetes mellitus. Med Clin (Barc). 2004; 122:96-8. 
